\title{
Comment on: "Evaluation of Neutrophil to Lymphocyte Ratio, Platelet to Lymphocyte Ratio, and Lymphocyte to Monocyte Ratio in Patients with Cellulitis"
}

DHasan Tahsin Gozdas ${ }^{1}$

1. Department of Infectious Diseases and Clinical Microbiology, Abant Izzet Baysal University Faculty of Medicine, Bolu, Turkey

Easily accessible hemogram-derived parameters have gained the attention of physicians recently. These are simple, practical, and economical parameters that were found useful in the prediction of many clinical problems. They are often used as a sign of chronic inflammation. In chronic inflammatory conditions, their levels are elevated. Chronic inflammation has been linked with the pathogenesis of many different diseases and their complications. Neutrophil to lymphocyte ratio (NLR) is one of the most important and popular hemogram biomarkers showing chronic inflammation.

Skin and soft tissue infections are common health problems in the community. Their symptoms may be similar to that of non-infective dermatological diseases. So, it is important to distinguish these infections from non-infective conditions to reach an early and accurate diagnosis and determine the treatment. Moreover, infection symptoms related to skin and soft tissue may be subtle in the geriatric population. So, it can be difficult to recognize these infections in this ever-increasing patient group.

In the article by Ince et al. ${ }^{1}$, NLR elevation was found to be beneficial in differentiating cellulite patients from non-infective healthy individuals. The authors also highlighted that NLR elevation was more evident in cellulite patients older than 65 years. So, NLR is a simple, practical, and economical hemogram biomarker that can assist us in the management of cellulite patients. Since the higher NLR levels are observed in patients over 65 years, this may be used as a clue to reach a diagnosis in this patient group. Future studies will provide us with new perspectives about the clinical use of NLR.

\section{REFERENCE}

1. Ince N, Güçlü E, Sungur MA, Karabay O. Evaluation of neutrophil to lymphocyte ratio, platelet to lymphocyte ratio, and lymphocyte to monocyte ratio in patients with cellulitis. Rev Assoc Med Bras. 2020;66(8):1077-1081.

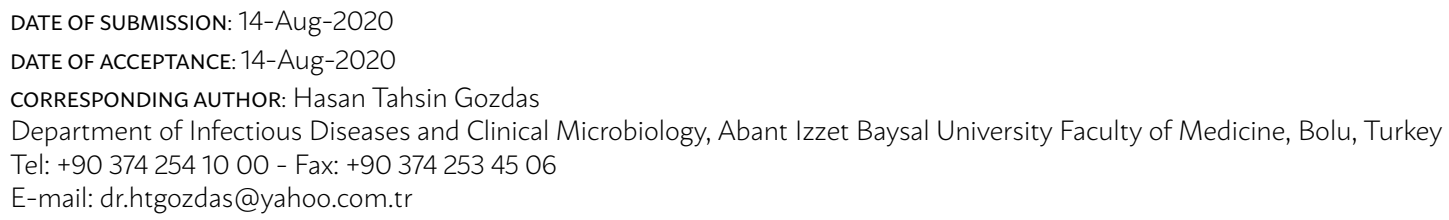

